\title{
A patterned macular dystrophy with yellow plaques and atrophic changes
}

\author{
PAUL CORTIN, DESMOND ARCHER, IRENE H. MAUMENEE, \\ KATHY FEIOCK, AND PERRY SPEROS
}

From the Wilmer Institute, Baltimore, Maryland, and the Department of Ophthalmology, Queen's University, Belfast

SUMMARY Three middle-aged male patients are described with a peculiar patterned dystrophy of the macula. The basic lesions are discrete yellow plaques typically confined to the macular area and radiating from the fovea. They appear to be located at the level of the retinal pigment epithelium (RPE). With the passage of time some of the yellow plaques altered in extent and configuration, and atrophic changes appeared or extended. Visual acuity and electrophysiological tests are either normal or only moderately affected. The lesions appear to be distinct from the patterned dystrophies of the retina already described and from other conditions characterised by yellow or white deposits at the level of the RPE.

Several varieties of patterned dystrophy of the retinal pigment epithelium (RPE) have been described. They include Sjögren's reticular dystrophy of the retinal pigment epithelium, ${ }^{1,2}$ macroreticular dystrophy of the retinal pigment epithelium, ${ }^{3}$ and butterfly-shaped pigment dystrophy of the fovea. ${ }^{4}$ The lesions are inherited, bilateral, and symmetrical and occur at the level of the RPE. The patterned dystrophies of the RPE do not significantly alter visual functions, and psychophysical and electrophysiological tests are generally only slightly affected.

Recently macroreticular, butterfly-shaped, and possibly reticular dystrophy of the RPE have all been observed in one family, suggesting a link between the patterned dystrophies of the RPE. ${ }^{5}$ This report describes 3 patients with an unusual bilateral and symmetrical dystrophy of the macular region characterised by discrete, yellow, plaque-like lesions at the level of the RPE and central atrophic changes of the outer retina.

\section{Case reports}

\section{PATIENT 1}

A 57-year-old male was seen by one of us (D.A.) in February, 1974 with a 6-month history of deterioration of vision in his left eye. He noticed that the 'middle of the word disappeared' when reading

Correspondence to Dr P. Cortin, Ophtalmologie, CHUL, 2705, boul. Laurier, Ste-Foy, Quebec, Canada, G1V 4G2. with his left eye. The patient had no history of previous eye disease or defective night vision, and no member of his family had any significant eye complaints.

On examination the best corrected visual acuity was $6 / 6$ right eye and 6/18 left eye. The anterior segments of the eyes were normal apart from an unusual vascular malformation of the right iris, which was considered an incidental finding. Each fundus showed oval or oblong, yellow-white plaques arranged about the macula in a spoke-like configuration often impinging on the fovea (Fig. 1).

The lesions were relatively discrete and associated only with subtle proliferative and atrophic changes. in the nearby RPE. Fundus biomicroscopy and stereoscopic photography indicated that the plaquelike lesions were slightly raised and located at the level of the RPE. There was no evidence of inflammation of the retina, choroid, or posterior vitreous.

The left eye showed multiple, small, discrete, white deposits within a central circular zone of pigment epithelial atrophy at the fovea. Several yellow-white plaques radiated from the central atrophic hub (Fig. 2). Fluorescein angiography of both posterior fundi (Figs. 3, 4) showed clearly defined areas of hyperfluorescence consistent with an abnormality of the RPE. The fluorescent defects. were more widespread than would have been predicted from ophthalmoscopy. The discrete yellow-white plaques remained hypofluorescent for the most part throughout angiography, and, although some staining of the adjacent RPE was 


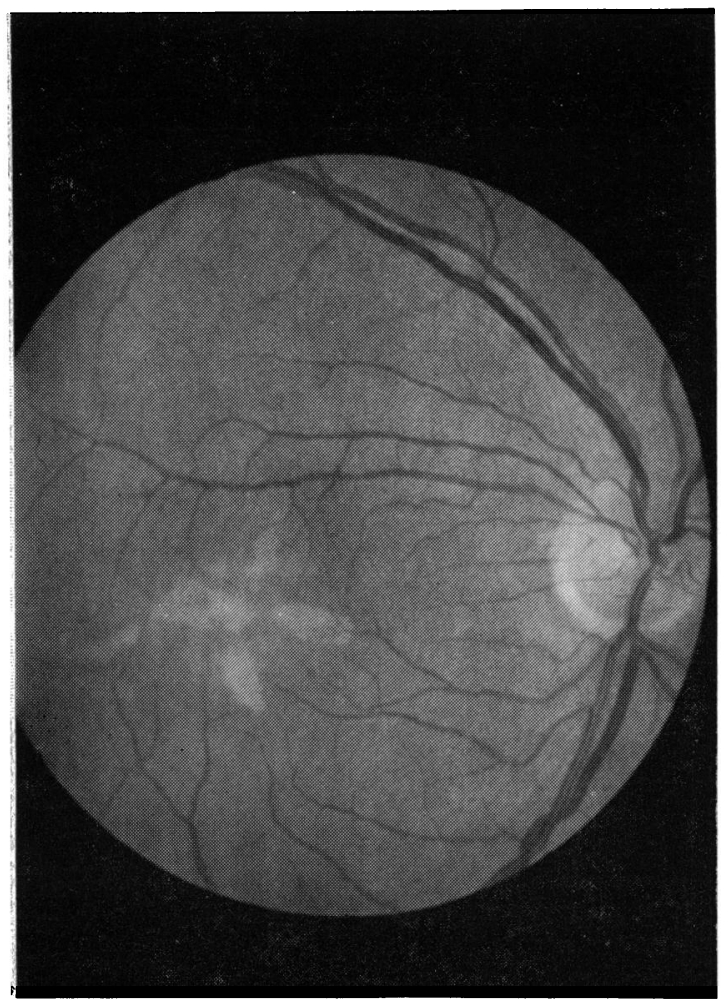

Fig. 1 Photograph right fundus, patient 1 (1974).

Discrete plaques measuring one-third to one-half disc diameter $(D D)$ in size radiate from the foveal region.

obvious in the late phases of angiography, there was no evidence of dye extravasation beyond the retinal pigment epithelial barrier into the outer retina.

Punctate hyperfluorescent defects were also noted beyond the macula in each eye, indicating a more widespread involvement of the RPE.

Static and kinetic perimetry (Tübinger) revealed a relative central scotoma in the right eye and a small absolute central scotoma in the left eye. The Farnsworth-Munsell 100 hue test revealed no specific colour vision abnormality, but the electrooculogram (EOG) was subnormal in each eye, i.e., 1.5 right and left (lower limit of normal is 1.8 for laboratory conditions). The photopic and scotopic electroretinographic responses were within normal limits. A genetic survey did not reveal any similar lesions in members of the family examined.

The fundus lesions were observed over a period of almost 4 years, during which time most of the oblong, yellow-white plaques either faded, diminished in size, or disappeared.

In one or two areas the plaques extended or

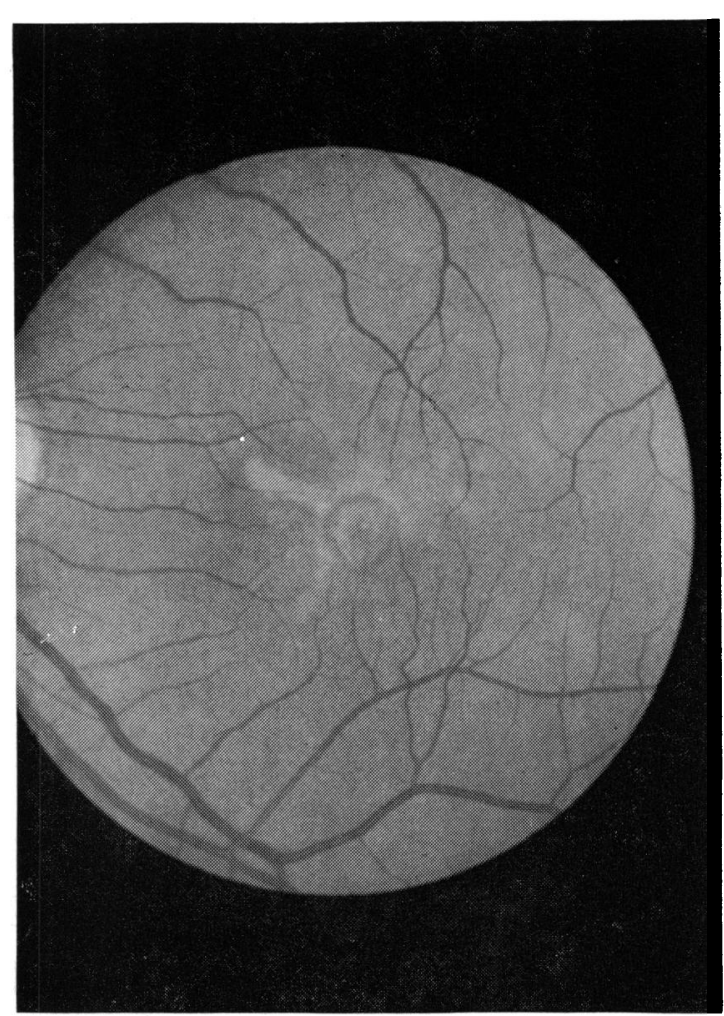

Fig. 2 Photography left fundus, patient 1 (1974).

Plaque-like lesions are arranged about the central circular area of pigment epithelial atrophy.

increased in size and in 1 eye several fresh, white deposits occurred within and superior to the macula (Fig. 5, arrows). The new plaques were morphologically and angiographically identical to those initially noted 4 years previously. An atrophic $\frac{0}{3}$ circular lesion of the RPE developed at the right fovea and intensified and increased in size at the $O$ left fovea (Fig. 6). Fluorescein angiography showed a slight increase in the area of defective pigment $\stackrel{\circ}{\triangle}$ epithelium at the macula, but there was no evidence $\frac{D}{0}$ of fluid accumulation either in the subpigment epithelial or subretinal spaces. Visual fields showed ${ }^{N}$ some intensification of the central and paracentral $N$ scotomata in each eye, though visual acuity re- $N$ mained $6 / 6$ right and $6 / 18$ left. Electrophysiological ${ }^{\omega}$ tests revealed no progression of the functional abnormalities.

PATIENT 2

A 41-year-old male was referred to one of us (D.A.) $\frac{0}{0}$ in February 1975 complaining of slight deterioration $\stackrel{\mathbb{D}}{\mathbb{D}}$ of vision in both eyes and in particular the left eye. $\stackrel{\stackrel{P}{\mathbb{D}}}{\mathrm{C}}$

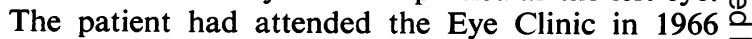




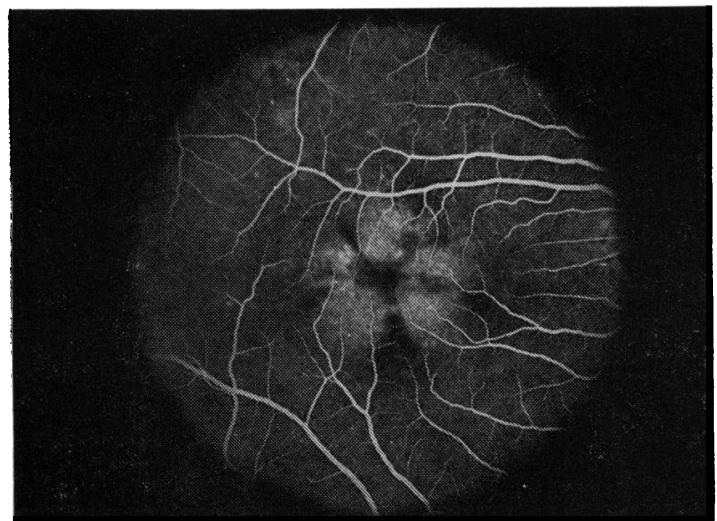

Fig. 3 Venous phase fluorescein angiogram of fundus shown Fig. 1. There is a discrete abnormality of the retinal pigment epithelium involving the macula. The plaque-like lesions are mostly hypofluorescent.

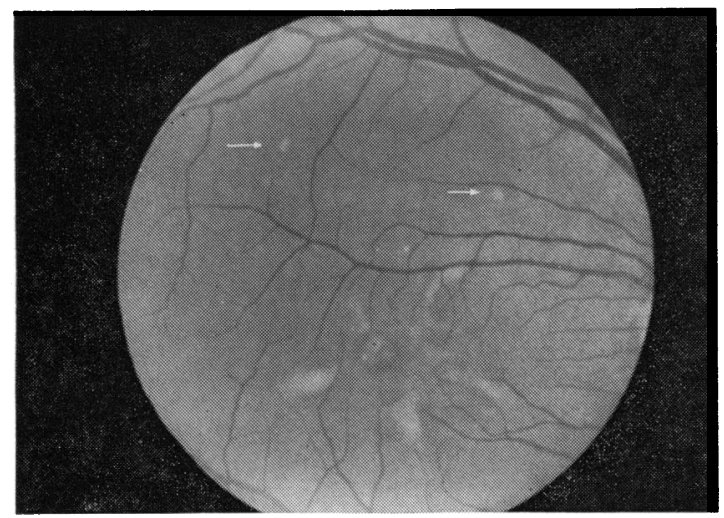

Fig. 5 Photograph right fundus patient 1 (1977). Some of the plaque-like lesions have faded, others have extended. Fresh lesions have occurred above the macula (arrows).

with recurrent attacks of bilateral episcleritis, and visual acuity at that time was recorded as $6 / 5$ right and left. The patient gave no history of defective night vision and was in good general health.

On examination the best corrected visual acuity was 6/9 right and 6/12 left. Static and kinetic Tübinger visual fields showed no abnormality, and colour vision was normal by the FarnsworthMunsell 100 hue test. The EOG was normal in the right eye $(2 \cdot 0)$ but significantly reduced in the left eye $(1 \cdot 17)$. Photopic and scotopic electroretinographic recordings were within normal limits for each eye.

Both fundi showed circular or oblong, yellowwhite plaques at the macula (Figs. 7,8 ) situated at the level of the RPE. There were scattered areas of retinal pigment epithelial atrophy and proliferation

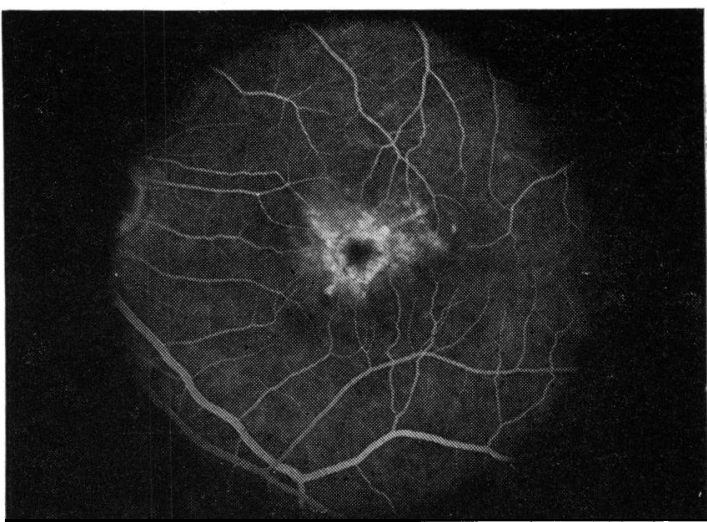

Fig. 4 Late phase fluorescein angiogram of fundus shown Fig. 2. The macula shows a mottled hyperfluorescence with some staining of the retinal pigment epithelial layer.

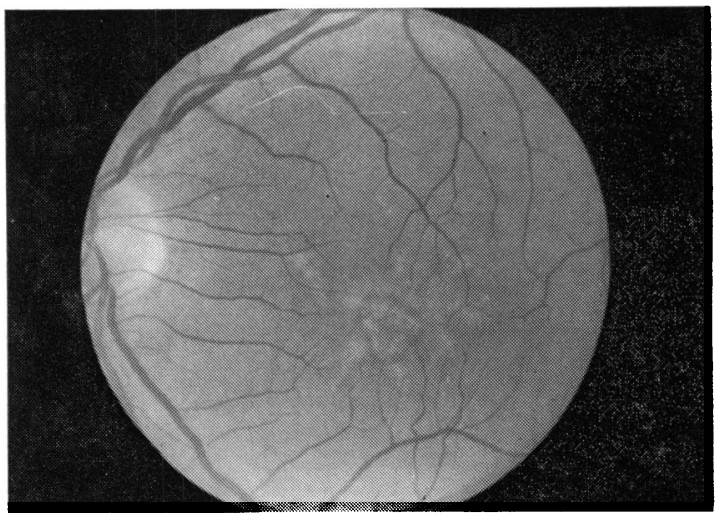

Fig. 6 Photograph left fundus, patient 1 (1977). The plaque-like lesions have faded and the central circular atrophic area has increased in size.

at and near some of the plaques and a well-defined, discrete circular zone of pigment epithelial atrophy at each fovea. Fluorescein angiography confirmed the presence of a retinal pigment epithelial abnormality at and surrounding each fovea. The dense yellow-white plaques and foci of pigment accumulation were hypofluorescent, and areas of pigment epithelial atrophy were hyperfluorescent (Figs. 9, 10).

Over a period of $2 \frac{1}{2}$ years the yellow-white lesions became less distinct and the central circular zone of retinal pigment epithelial atrophy became more marked. Fluorescein angiography did not indicate any significant increase in the extent of the lesion, which remained confined to the macula. A survey of the patient's family revealed no characteristic macular lesions; however, 1 brother had extensive drusen of both fundi, a widespread atrophy 
Fig. 7 Photograph right fundus, patient 2 (1975).

Faint white plaques surround a circular zone of pigment epithelial atrophy.

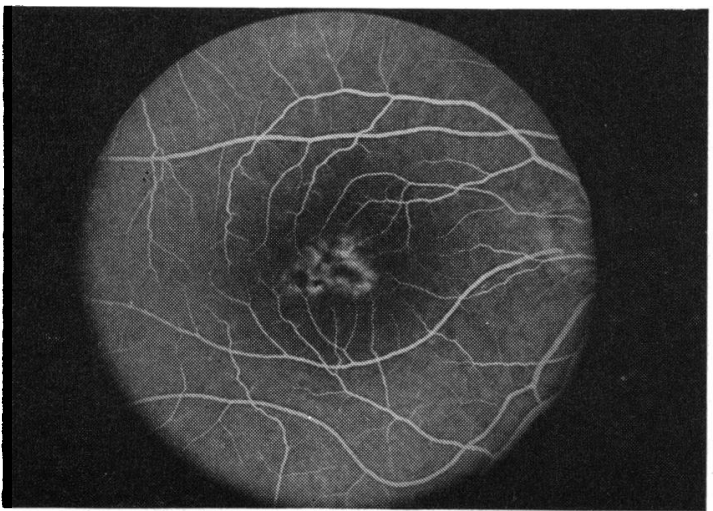

Fig. 9 Venous phase fuorescein angiogram of fundus shown Fig. 7. Discrete pigment epithelial defects fluoresce brightly.

of the RPE, and a visual acuity diminished to $6 / 24$ right and 3/60 left. The patient's father also had poor vision and was able to read only large print using a magnifying glass from the age of 60 . The patient's father and mother were first cousins.

\section{PATIENT 3}

A 52-year-old male was referred to one of us (I.H.M.) in April 1974. He had noticed about the age of 40 that vision had decreased in each eye. During the 3 years prior to presentation, he had been treated for asthma with prednisone $5 \mathrm{mg}$ per day and for kidney stones with allopurinol. On examination the best visual acuity was 6/18 right and 6/12 left. Routine eye examination was normal apart from the changes noted on funduscopy. The right fundus (Fig. 11) showed a pigmented ring of less than 1 disc diameter (DD), within which a thinned retina and choroidal vessels were seen

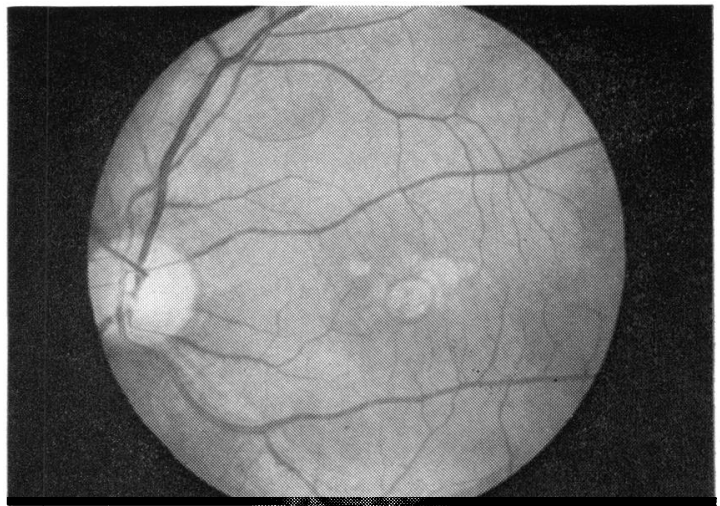

Fig. 8 Photograph left fundus, patient 2 (1975). Well defined white plaque-like lesions radiate from a central circular area of pigment epithelial atrophy.

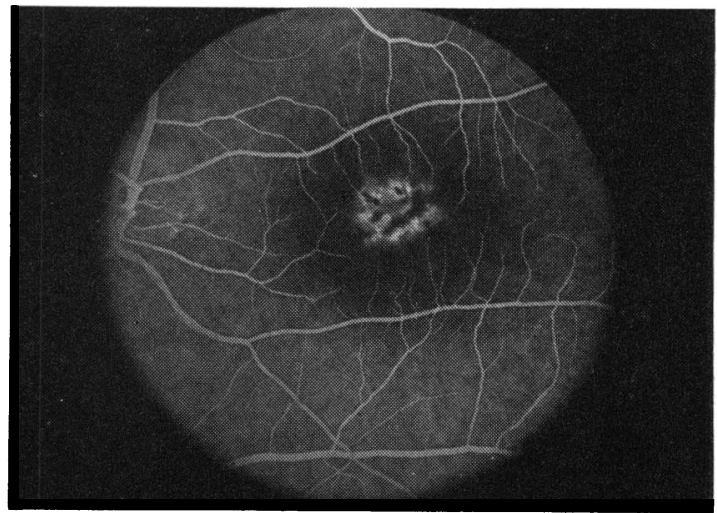

Fig. 10 Late-phase fluorescein angiogram of fundus shown Fig. 8. The plaque-like lesions are mostly hypofluorescent and there is some staining of the affected retinal pigment epithelial layer.

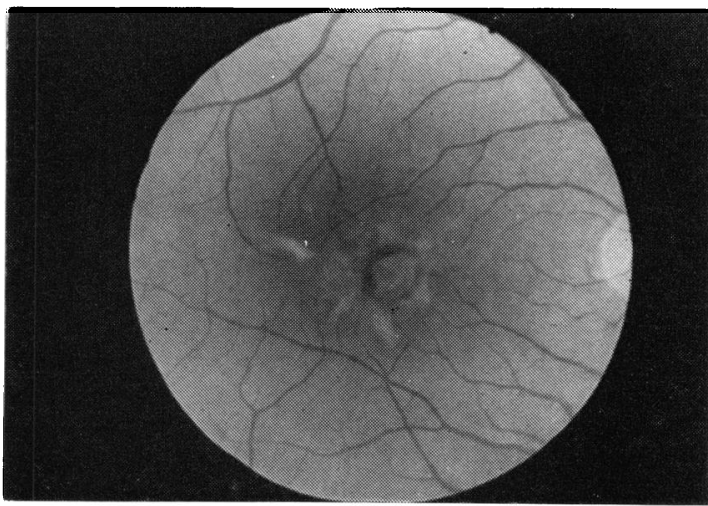

Fig. 11 Fundus photograph right eye, patient 3. A pigmentary ring delineates a central area of retinal pigment epithelial atrophy. Yellow stripes are radially arranged around the atrophic zone. 


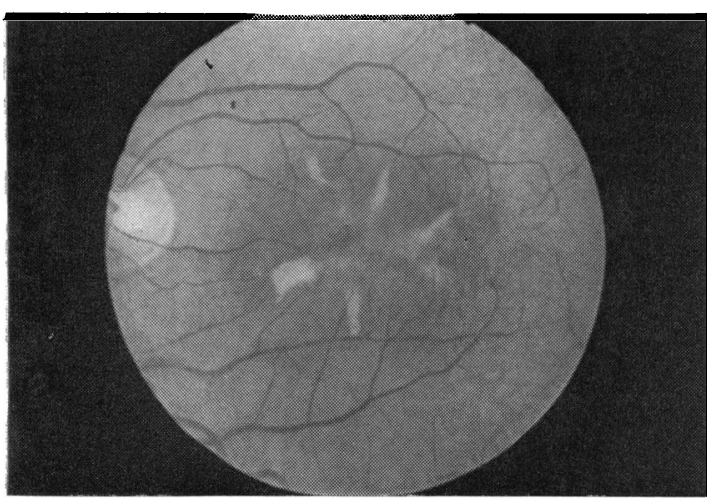

Fig. 12 Left fundus photograph, patient 3. Radiating yellow stripes are arranged around the fovea in $a$ petaloid fashion.

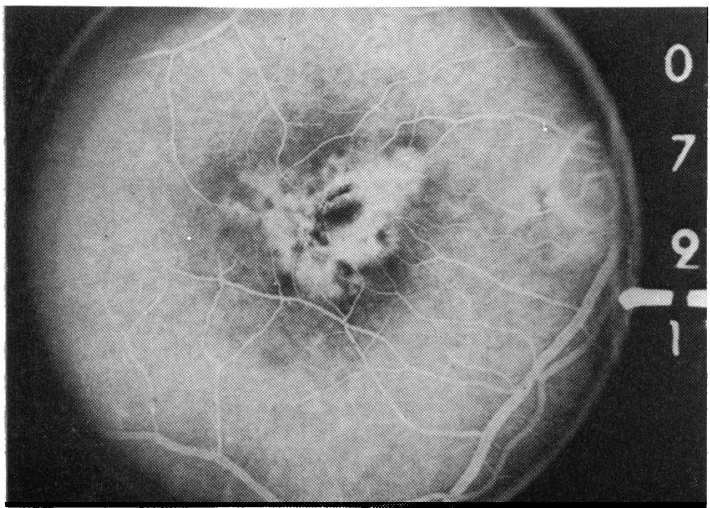

Fig. 14 Late phase fluorescein angiogram of fundus shown in Fig. 11. The yellow stripes are hypofluorescent and are highlighted against the hyperfluorescent pigment epithelial defects.

with the slit-lamp. Around the pigmented ring the retina showed a greyish yellow material deposited in a radial fashion deep in the retina or at the level of the RPE.

The left macula (Fig. 12) also demonstrated yellowish deep-seated deposits which were more distinct than those noted in the right fundus. The abnormal material radiated in a petaloid fashion from the region of the left fovea. There was no evidence of microcystoid degenerative changes. Over a period of 20 months 1 of the deposits (noted at 11 o'clock) partially faded, leaving a deep-seated pigmentary line (Fig. 13).

During fluorescein angiography the right macula (Fig. 14) showed a central dark zone within the pigment ring noted on ophthalmoscopy. This, in view of the biomicroscopic findings, suggested a

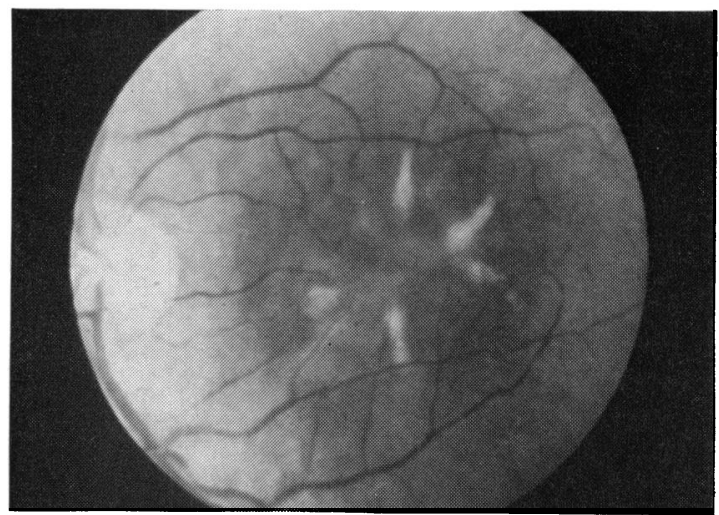

Fig. 13 Left fundus photograph, patient 3, taken 20 months after fundus photograph shown in Fig. 12. The 11 o'clock stripe has faded leaving a pigmentary line. The 4 o'clock stripe may have extended (some artefacts are present).

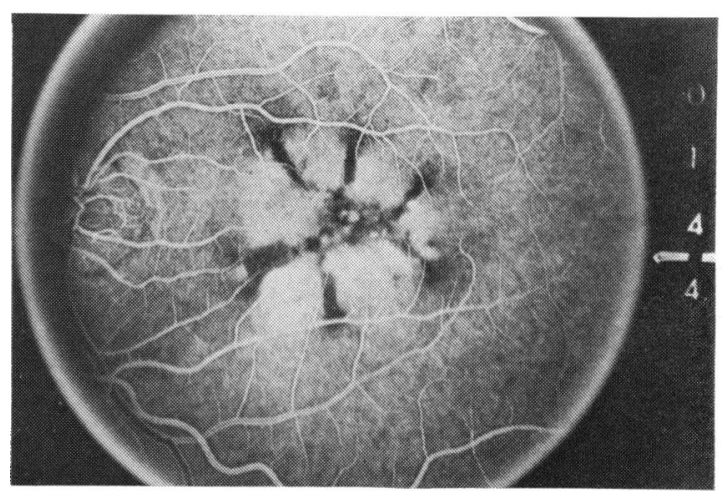

Fig. 15 Early venous phase angiogram of fundus shown in Fig. 12. The yellow stripes are strikingly hypofluorescent or nonfluorescent.

localised atrophy of the choriocapillaris. Peripheral to the ring a $10^{\circ}$ wide circle of hyperfluorescence was evident. Stripes of hypofluorescence corresponded more or less to the yellow deposits highlighted on the hyperfluorescent background. On the left side (Fig. 15) fluorescein angiography showed six nonfluorescent, radially orientated stripes contrasting against the hyperfluorescent background.

Patient 3's 54-year-old only brother noticed decreased vision in his left eye at age 26 . Vision is thought to have been stable since. There was no metamorphopsia or photophobia; however, the patient admitted to some degree of night blindness. Routine ocular examination showed no structural abnormalities apart from the lesions noted at the fundi. The corrected visual acuity was $6 / 7.5$ right and 6/36 left. The right fundus showed a small 


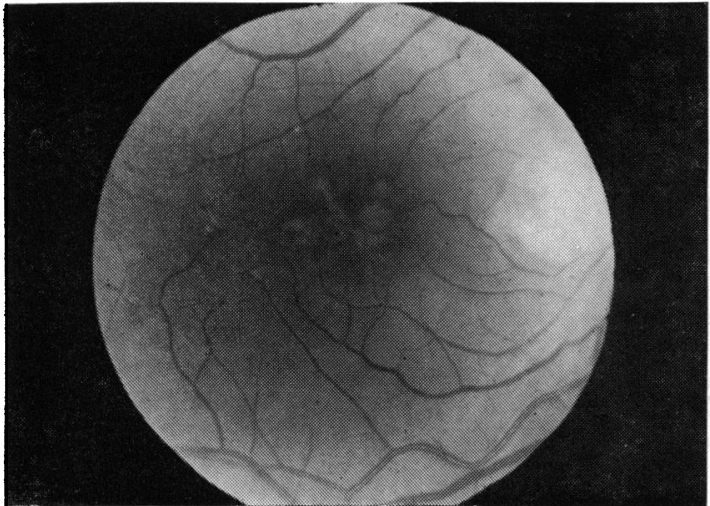

Fig. 16 Right fundus photograph of patient 3's brother. There is a small perifoveal atrophic area. Ill-defined yellow deposits are present and a zone of peripapillary atrophy is noted.

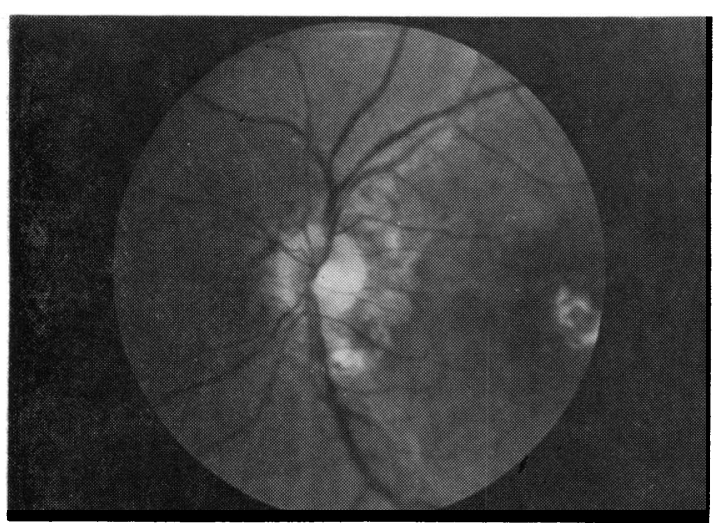

Fig. 18 Left fundus photograph patient 3's brother. There is marked peripapillary chorioretinal atrophy and a macular scar.

circular zone of retinal atrophy nasal to the fovea. There were some ill-defined stripes of yellow deposits which were hypofluorescent during fluorescein angiography (Figs. 16, 17). The left fundus showed an atrophic maculopathy with peripapillary choroidal atrophy and peripheral postequatorial pigmented lesions (Figs. 18, 19, 20), a picture reminiscent of the presumed ocular histoplasmosis syndrome. During angiography a focal area of hyperfluorescence was noted at the left macula indicating a defect of the retinal pigment epithelium (Fig. 20).

No abnormality was detected on routine examination of patient 3's three children except for 2 drusen at the right posterior fundus of his 16-yearold daughter (Fig. 21).

Patient 3's parents were not examined. His father was said to have normal vision, but his

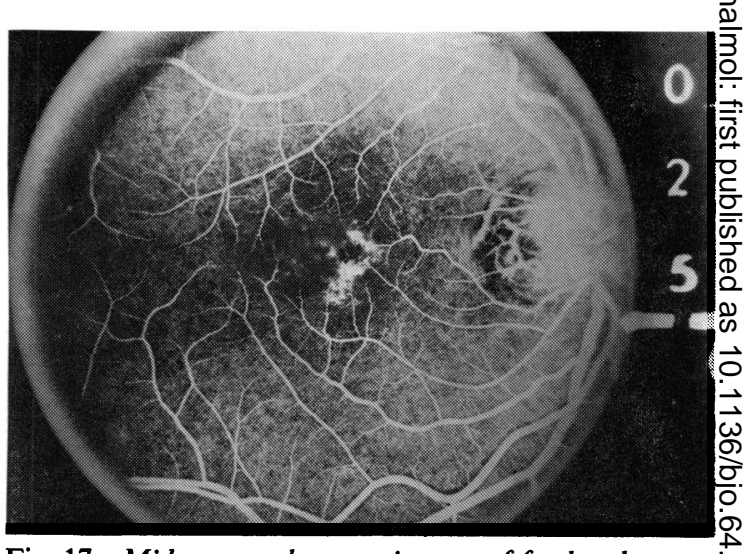

Fig. 17 Mid venous phase angiogram of fundus shown mid venous. There are discrete hyperfluorescent pigment epithelial defects. The yellow plaques are hypofluorescent.

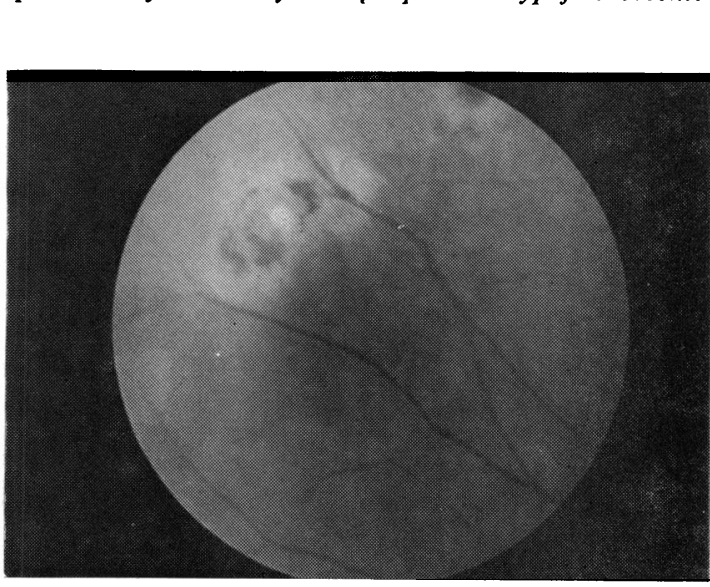

Fig. 19 Left superonasal peripheral fundus patient 3's brother. Postequatorial pigmented lesions are present.

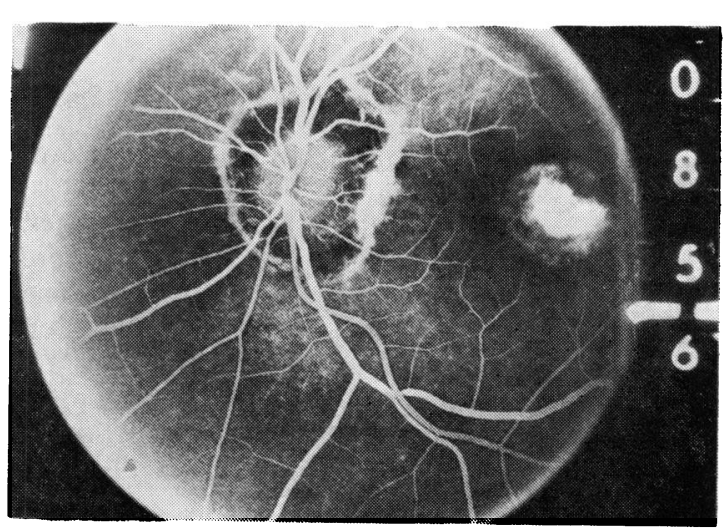

Fig. 20 Late venous phase angiogram of fundus shown mid venous. There is striking peripapillary and macular hyperfluorescence. The features are reminiscent of the presumed ocular histoplasmosis syndrome.

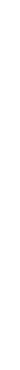




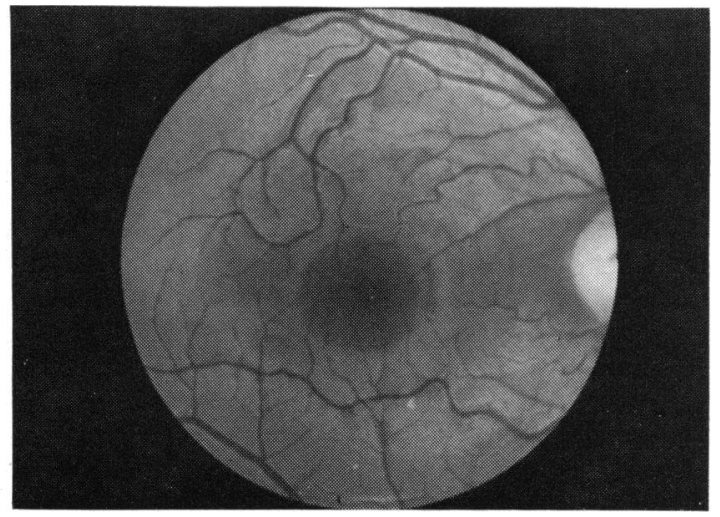

Fig. 21 Right fundus photograph of 16-year-old daughter of patient 3. Two small drusen are present below the macula.

mother used a magnifying glass in addition to her bifocals after the age of 60 .

Extensive psychophysical tests were performed by one of us (K.F.) on patient 3 , his brother, and his 3 children. The perimetric studies were done with the Tübinger and Ferre-Rand perimeters. Essentially, they showed central scotomas in the 2 brothers and relative parafoveal scotomas in patient 3's daughter. The dark adaptation curves with the Tübinger perimeter were somewhat subnormal in the 2 brothers, in accordance with the symptoms. Colour tests were within normal limits except for the left eye of the brother.

The ERGs were recorded by one of us (P.S.). A modified Rabin and Berson's technique ${ }^{6}$ was used. They showed reduced amplitudes and prolonged implicit times of both photopic and scotopic ERGs in patient 3 and his brother. ERGs were normal in the children. Normal EOG ratios were found in both brothers.

\section{Discussion}

Three patients with unusual but characteristically patterned lesions of the macula are presented. The patients are all male and suffered only mild, moderate, or no disturbance of visual functions. The exact age of onset of the lesions was difficult to establish, but it probably occurred between the ages of 30 and 50. The basic abnormality was an oval or oblong, yellow, plaque-like lesion confined to the macular area. The plaques were multiple, relatively discrete, about one-third to one-half a disc diameter in size, and typically arranged in a radial pattern about the fovea. Fundus biomicroscopy and stereophotography confirmed that the lesions were slightly elevated and located at the level of the RPE. The neighbouring retina showed a variety of RPE changes, some atrophic and some proliferative. The earliest lesions, which were usually asymptomatic, had a uniform yellow-white hue, but with time usually faded, disappeared, or were replaced by an area of RPE atrophy or proliferation. A circular area of atrophic retinal pigment epithelium characteristically developed at or near the fovea in all cases, and at this stage there was generally some abnormality of central visual function. Other changes included circular areas of retinal thinning and choriocapillaris loss. In 1 patient fresh macular and extramacular lesions developed over a period of 2 to 3 years. These additional lesions had identical characteristics to those plaques noted at initial examination.

The existence of some similar lesions in the right eye of patient 3's brother suggests that the condition may be familial, but it is not possible from the current data to establish a mode of inheritance. It it left to other workers in the future to be more explicit on the genetics, if indeed further cases would confirm our present strong suspicion that it is a heritable condition.

No histopathological studies have been undertaken of the lesions in question and their exact nature is not known. However, ophthalmoscopic and angiographic evidence suggest that the disease primarily affects the RPE, and the typical yellow plaques probably represent a deposition or accumulation of some substance within the RPE or in its immediate vicinity. Despite the discrete morphology of the individual plaques there is reason to suspect a diffuse abnormality of the RPE in some patients on account of the widespread abnormalities on fluorescein angiography and the significant EOG findings. The retinal receptors appear relatively unaffected, at least until late in the disease process.

The lesions described are distinctive and morphologically different from the other varieties of patterned dystrophy of the macula. The radially arranged yellow plaques are not typical of the reticular dystrophies or the butterfly dystrophy of the fovea, and no atrophic changes have been described in latter conditions. The yellow plaques resemble confluent drusen in that they occur at the level of the RPE, are discrete, and predominantly affect the posterior fundus. However, the yellow plaques show little tendency to enlarge concentrically and are characteristically hypofluorescent during angiography. The radial disposition of the subretinal plaques is quite different from the general distribution of drusen, and no obvious decompensation of the retinal pigment epithelial barrier or development of a disciform response has been noted.

The lesions characteristic of patterned dystrophy 
described in this report also differ fundamentally from those typically of fundus flavimaculatus in their hue, size, and distribution. The yellow patterned lesions show some of the characteristics of the deposits encountered in vitelliform macular degeneration (Best's disease). They are both yellow, situated at the level of the RPE, and associated with relatively mild changes in central visual functions. The appearance of fresh lesions at the posterior pole of the eye during the evolution of the yellow plaques may also occur in vitelliform macular degeneration. The patterned dystrophy, however, differs from vitelliform macular degeneration in that there is no evidence of a yolk-like lesion at any stage of the disease process or layering of the abnormal deposits. In addition the electrooculographic changes in vitelliform macular degeneration are more uniform and severe than those noted in our patients.

A peculiar foveomacular dystrophy described by Gass $^{2}$ shows some of the clinical characteristics of the patterned dystrophy reported in this paper. In both groups of patients the condition began between 30 and 50 years of age and affects vision relatively slightly. The lesions in both conditions are subretinal, symmetrical, and slowly progressive. The lesions described by Gass, however, are less regular, not disposed in a petaloid pattern, and have a uniform, central pigmented spot at the fovea. In addition many of his cases are also associated with a more widespread disturbance of the RPE and to some degree resemble hereditary drusen.

Singerman et al. ${ }^{8}$ have recently described a macular dystrophy in a large sibship and labelled the condition dominant slowly progressive macular dystrophy. Some of the patients described in this report have macular lesions resembling the yellow plaques described above and showed similar angiographic features. It may be that there is some overlap in the cases described in our report and certain of the members of their large sibship.

Although the patterned dystrophy with yellow plaques and atrophic changes resembles several well documented focal abnormalities of the RPE, it nevertheless has distinct morphological characteristics, a natural course and fundus findings, and it is probably justifiable to consider it as a separate clinical entity. The yellow plaques almost certainly relate to an abnormality or malfunction of the RPE and as such probably form a small part of the wide spectrum of conditions which reflect a malady of this highly specialised layer.

\section{References}

'Sjögren H. Dystrophia reticularis laminae pigmentosae retinae. Acta Ophthalmol 1950; 28:279-295.

${ }^{2}$ Deutman AF, Rumke AML. Reticular dystrophy of the retinal pigment epithelium. Arch Ophthalmol 1969; 82:4-9. ${ }^{3}$ Mesker RP, Oosterhuis JA, Delleman JW. A retinal lesion resembling Sjögren's dystrophia reticularis laminae pig mentosae retinae. In: Perspectives in Ophthalmology 2: 40-45. Amsterdam: Excerpta Medical Foundation, 1970.

${ }^{4}$ Deutman AF, van Blommestein JDA, Henkes HE, Waardenburg PJ, Solleveld-Van Driest E. Butterfly-shaped pigment dystrophy of the fovea. Arch Ophthalmol 1970;83 558-569.

${ }^{5}$ Hsieh RC, Fine BS, Lyons JS. Patterned dystrophies of the retinal pigment epithelium. Arch Ophthalmol 1977; 95 : 429-435.

${ }^{6}$ Rabin AR, Bearson EL. A full-field system for clinical electroretinography. Arch Ophthalmol 1974; 92:59-63.

${ }^{7}$ Gass JDM. A clinicopathologic study of a peculiar foveomacular dystrophy. Trans Am Ophthalmol Soc 1974; 72: 139-156.

${ }^{8}$ Singerman LJ, Berkow JW, Patz A. Dominant slowly progressive macular dystrophy. Am J Ophthalmol 1977 83:680-693. 